\title{
EFECTO DEL CONTROL DE MALEZAS CON PARAQUAT Y GLIFOSATO SOBRE LA EROSIÓN Y PÉRDIDA DE NUTRIMENTOS DEL SUELO EN CAFETO ${ }^{1}$
}

\author{
Robin Gómez Gómez ${ }^{2}$
}

\begin{abstract}
RESUMEN
Efecto del control de malezas con paraquat y glifosato sobre la erosión y pérdida de nutrimentos del suelo en cafeto. En Alajuela, Costa Rica, se realizó un experimento en los años 2002 y 2003 tendiente a determinar el control de malezas, la dinámica poblacional de las malezas y la erosión y pérdida de nutrimentos del suelo debido a aplicaciones sucesivas de paraquat y glifosato. Dos parcelas de $900 \mathrm{~m}^{2}$ dentro de una plantación de café fueron aplicadas con paraquat y dos más tratadas con glifosato. En el año 2003 una parcela de cada tratamiento fue disturbada aplicándole materia orgánica (broza de café) y carbonato de calcio. El agua de escorrentía y los sedimentos provenientes de parcelas de $2 \times 7 \mathrm{~m}$ fueron recogidos en colectores de 200 litros de capacidad y se midieron las pérdidas de nutrientes. Al inicio de los tratamientos el control de malezas con ambos herbicidas fue de $90-100 \%$, posteriormente con las continuas aplicaciones de herbicidas, se presentaron malezas tolerantes, haciendo necesario el control mecánico y aumentar la dosis del herbicida para controlarlas. Durante el 2002 las pérdidas de suelo de las parcelas tratadas con glifosato fueron el doble $(3,956 \mathrm{t} / \mathrm{ha})$ comparadas con las ocurridas en las parcelas aplicadas con paraquat (1,936 t/ha). En el 2003, en las parcelas no disturbadas tratadas con paraquat se determinó menos erosión (0,231 t/ha) en comparación con 0,329 t/ha en aquellas aplicadas con glifosato. Asimismo, en las parcelas disturbadas por enmiendas las pérdidas fueron menores en las aplicadas con paraquat: $0,170 \mathrm{vs.} 0,187 \mathrm{t} / \mathrm{ha}$ de glifosato. Los análisis de sedimentos y agua mostraron una pérdida importante de $\mathrm{Ca}, \mathrm{Mg}$ y $\mathrm{K}$, similar en ambos tratamientos. El rendimiento estimado en el año 2003 fue muy similar en ambos tratamientos.
\end{abstract}

Palabras claves: erosión del suelo, paraquat, glifosato, control de malezas, pérdida de nutrimentos.

\begin{abstract}
Effects of weed control with paraquat and glyphosate on erosion and soil nutrient losses in coffee. A trial was conducted during 2002-2003 at Alajuela, Costa Rica to determine weed control, weed dynamics, soil erosion and nutrient losses due to successive applications of paraquat and glyphosate. Two plots of $900 \mathrm{~m}^{2}$ were treated with paraquat and two more plots were treated with glyphosate. During 2003 one plot of each treatment was ammended with calcium carbonate and organic mater (shell of coffee berry). Run-off water and sediments from a $2 \times 7 \mathrm{~m}$ plot were collected into a 2001 drum. The amount of nutrient lost from each plot was determined. The first year both treatments provided 90-100\% of weed control but tolerant weeds were selected. A mechanical control and an increase of the herbicides application rate were necessary to keep these weeds under control. During 2002 soil losses from glyphosate treatments were double (3.956 MT/ha) compared to that occurred from the paraquat treated areas (1.936 MT/ha). During 2003 at the amended plots again areas treated with paraquat had less erosion $(0,231 \mathrm{MT} / \mathrm{ha})$ vs $0,329 \mathrm{MT} / \mathrm{ha}$ from glyphosate sprayed plots. Soil losses from the amended plots were 0,170 $\mathrm{MT} / \mathrm{ha}$ and $0,187 \mathrm{MT} / \mathrm{ha}$, respectively. Sediment analysis indicates that $\mathrm{Ca}, \mathrm{Mg} \& \mathrm{~K}$ losses were similar in both treatments. In 2003 the estimated yield was similar in both areas.
\end{abstract}

Key words: soil erosion, glyphosate, weed control, nutrient loss.

\section{INTRODUCCIÓN}

En Costa Rica, al igual que en varios otros países cafetaleros de la región, la mayoría de las plantaciones de café se encuentran en zonas de ladera, con pendientes de hasta un $60 \%$ y en algunos casos de gran longitud. Lo anterior provoca que el agua de lluvia, por medio del salpique y la escorrentía, erosione estos suelos

1 Recibido: 8 de setiembre, 2004. Aceptado: 18 de enero, 2005.

2 Estación Experimental Fabio Baudrit Moreno, Universidad de Costa Rica. Correo electrónico: rgomezg@cariari.ucr.ac.cr. 
y disminuya la cantidad de bases en los mismos, haciéndolos a su vez menos fértiles y aumentando su acidez (Bertsch 1995; Barbier y Bishop 1995; Reynaud et al. 2002; Bertol et al. 2003). Este proceso puede afectar el rendimiento del cafetal, aumentar los gastos por concepto de fertilizantes y enmiendas y provocar una mayor susceptibilidad de las plantas a las enfermedades y plagas de insectos. Todo lo anterior afecta negativamente los ingresos del caficultor y su familia.

Desde el punto de vista de la agricultura, tanto la erosión como la sedimentación afectan la productividad del suelo (Barbier y Bishop 1995). La erosión disminuye la productividad del suelo al disminuir la profundidad de las raíces y también las reservas de agua y nutrientes, mientras que la sedimentación tiene serias implicaciones ambientales y económicas al disminuir la capacidad de los reservorios y atascar los canales de irrigación (Lal 1990; Hansen et al. 2002; Cogo et al. 2003).

Nearing et al. (2004) sostienen que el fenómeno del calentamiento global provocaría un ciclo hidrológico más fuerte, lo que conlleva a un mayor volumen, frecuencia e intensidad de las precipitaciones. Estos cambios en la lluvia, junto con cambios en temperatura, radiación solar, y concentración de $\mathrm{CO}_{2}$, tendrían impactos significativos en las tasas de erosión del suelo.

Algunos productores de café recurren a prácticas de conservación de suelos, tales como gavetas, canales para corte de aguas de escorrentía, barreras con materiales de poda y coberturas con algunas especies cultivadas o bien con malezas nobles como Arachis pintoi entre otras (Aguilar et al. 2003).

El control total de malezas dejando el suelo desnudo durante la época lluviosa y en cafetales nuevos, puede favorecer aún más la erosión del suelo, ya que recibe la mayor parte de la energía cinética de la precipitación, quebrando los agregados. Con la destrucción de los agregados, las partículas menores penetran y obstruyen los poros, disminuyendo la permeabilidad y formando un sello superficial o "costra", influenciando la infiltración del agua en el suelo (Reynaud et al. 2002).

En las plantaciones convencionales de café en Costa Rica, es común el uso de paraquat o bien de glifosato en aplicaciones solas o en mezclas con herbicidas de efecto residual como la terbutilazina y el oxifluorfén. El glifosato afecta y produce la muerte tanto de la parte aérea como del sistema radical de la planta, ya que su acción es sistémica y es traslocado a toda la planta. Por su parte, el paraquat afecta solamente la parte aérea que ha tenido contacto con el producto, traslocándose muy poco dentro de la planta (Vencill 2002). Esta diferencia en su modo de acción podría eventualmente retrasar el proceso de erosión cuando las raíces permanecen en el suelo por un periodo de tiempo mayor, contribuyendo con la unión de las partículas del mismo, mejorando la estructura del suelo, la aireación y la infiltración del agua en el suelo.

Esta investigación se realizó con la finalidad de estudiar el cambio que se produce en la población de malezas en una plantación de café joven después de realizar aplicaciones sucesivas de los herbicidas paraquat y glifosato, además de comparar la cantidad de suelo y nutrimentos que se pierden en el agua de escorrentía de las parcelas de café tratadas con estos herbicidas.

\section{MATERIALES Y MÉTODOS}

Se establecieron a inicios del año 2002 cuatro parcelas de $900 \mathrm{~m}^{2}$ cada una, separadas $1,5 \mathrm{~m}$ entre sí. Dentro de cada una de ellas se ubicó una "parcela de escorrentía" de 2 por 7 metros delimitada por láminas galvanizadas, donde se determinó la erosión y escorrentía.

La plantación de café donde se instalaron estas parcelas de investigación se ubica en la finca Montecristo, en San Isidro de Alajuela, Costa Rica, a $1.505 \mathrm{msnm}$, $10^{\circ} 098^{\prime}$ latitud norte, $84^{\circ} 189^{\prime}$ longitud oeste. Las plantas de café variedad catuaí tenían dos años de establecidas, con dos ejes ortotrópicos, a una distancia entre plantas de 0,90 metros y 1,80 entre hileras en el momento de iniciar las mediciones.

En las aplicaciones de los herbicidas se utilizó un equipo de mochila Spray Mec de 16 litros, con una boquilla de abanico plano Tee Jet XR8001 para las aplicaciones de glifosato y XR8003 para las de paraquat. La presión fue de $29 \mathrm{lbs} /$ pulgada $^{2}$ controlada por un regulador de presión acoplado al equipo de aplicación. El volumen de aplicación varió entre 153 y 200 1/ha al aplicar glifosato y 300-355 1/ha en las aplicaciones de paraquat, debido a que se buscó mejorar el control de ambos herbicidas sobre las malezas con cierto grado de tolerancia. Las aplicaciones se realizaron cuando las parcelas tuvieron una cobertura de malezas de un 30$40 \%$. En las parcelas 1 y 3 se aplicó paraquat (Gramoxone 20SL), y en las parcelas 2 y 4 se aplicó glifosato (Roundup 35,6SL). El Cuadro 1 muestra el detalle de las dosis y fechas de las actividades relacionadas con el control de malezas, realizadas en el área experimental. No se utilizó diseño estadístico. 
Hacia finales del año 2002, semana 38, fue necesario recurrir a una chapia con motoguadaña (cortadora de motor) en las parcelas 1 y 3 , para controlar tres especies tolerantes al paraquat: Gnaphalium sp., Conyza sp. y Hydrocotyle bowlesioides. Por otro lado, en las parcelas tratadas con glifosato fue necesario aplicar $1.088 \mathrm{gia} / \mathrm{ha}$ en la última aplicación de este año, ya que en estas parcelas se seleccionaron malezas de la familia de las Rubiáceas, tolerantes al glifosato, cuyo control fue imposible con la dosis utilizada inicialmente (712 gia/ha). En el año 2003 se recurrió nuevamente al control con motoguadaña en las parcelas tratadas con paraquat y al uso del glifosato más concentrado en la parcela 4, para el control de tres especies del género Spermacoce: S. latifolia, S. ocimoides y S. laevis, además de hacer una chapia con cuchillo.

A inicios del año 2003 se introdujo un nuevo tratamiento, el cual consistió en la aplicación de carbonato de calcio y materia orgánica -broza o cáscara de café- al suelo e incorporarla, disturbando el suelo. Este tratamiento se realizó en dos parcelas, una de paraquat y la otra de glifosato (parcelas 3 y 4). Esto hizo que se mo- dificaran características físicas del suelo y por lo tanto se afectó la escorrentía y erosión en las "parcelas de escorrentía" ya establecidas. La introducción de este tratamiento se debió a que ésta es una labor normal dentro de la finca cuando los análisis del suelo indican que existen problemas de acidez y deficiencia de materia orgánica.

Las variables evaluadas fueron:

\section{Control químico de las malezas:}

Se realizaron evaluaciones semanales después de la aplicación de los herbicidas en estudio, donde se determinó el porcentaje de control sobre las malezas usando la escala propuesta por Camper (1986). Asimismo, se realizaron levantamientos de malezas a lo largo del periodo de evaluación para establecer el cambio en la población de éstas. Se utilizó el Indice de Frecuencia Cobertura (IFC) para analizar cuáles fueron las malezas predominantes o más importantes a lo largo del año en cada parcela por separado. Se determinó el IFC según la metodología propuesta por Jürgens (1985).

Cuadro 1. Fecha (semana del año) y descripción de las aplicaciones de herbicidas y técnicas utilizadas en el control de malezas en las parcelas en estudio. San Isidro, Alajuela, Costa Rica, 2002 y 2003.

\begin{tabular}{|c|c|c|c|c|c|}
\hline \multicolumn{3}{|c|}{ Año 2002} & \multicolumn{2}{|r|}{ Año 2003} & \multirow[b]{2}{*}{ Parcela } \\
\hline Sema & Descripción & Parcela & Semana & Descripción & \\
\hline 3 & Paraquat $500 \mathrm{~g} / \mathrm{ha}$ (volumen de & $1 \times 3$ & & Chania con motoouadaña & $1 \times 3$ \\
\hline 26 & $\begin{array}{l}\text { aplicación: } 300 \mathrm{~L} / \mathrm{ha}) \\
\text { Paraquat } 500 \mathrm{~g} / \mathrm{ha} / \mathrm{ha}(300 \mathrm{l} / \mathrm{ha})\end{array}$ & $\begin{array}{l}1 \text { y } 3 \\
1 \text { y } 3\end{array}$ & $\begin{array}{r}3 \\
20\end{array}$ & $\begin{array}{l}\text { Chapia con motoguadaña } \\
\text { Paraquat } 500 \mathrm{~g} / \mathrm{ha}(318 \mathrm{l} / \mathrm{ha}) \text {, }\end{array}$ & $\begin{array}{ll}1 \\
\text { y } 3 \\
1\end{array}$ \\
\hline 26 & Glifosato $712 \mathrm{~g} / \mathrm{ha}(200 \mathrm{l} / \mathrm{ha})$ & 2 y 4 & 20 & Glifosato $712 \mathrm{~g} / \mathrm{ha}(153 \mathrm{l} / \mathrm{ha})$ & 2 \\
\hline 33 & Paraquat $500 \mathrm{~g} / \mathrm{ha}(300 \mathrm{l} / \mathrm{ha})$ & 1 y 3 & 23 & Paraquat $500 \mathrm{~g} / \mathrm{ha}(318 \mathrm{l} / \mathrm{ha})$, & 3 \\
\hline 36 & Paraquat $500 \mathrm{~g} / \mathrm{ha}(300 \mathrm{l} / \mathrm{ha})$ & 1 y 3 & 23 & Glifosato $712 \mathrm{~g} / \mathrm{ha}(153 \mathrm{l} / \mathrm{ha})$ & 4 \\
\hline 37 & Glifosato $712 \mathrm{~g} / \mathrm{ha}(200 \mathrm{l} / \mathrm{ha})$ & 2 y 4 & 28 & Paraquat $500 \mathrm{~g} / \mathrm{ha}(355 \mathrm{l} / \mathrm{ha})$, & 1 \\
\hline 38 & Chapia con motogüadaña & 1 y 3 & 28 & Glifosato $1088 \mathrm{~g} / \mathrm{ha}(166 \mathrm{l} / \mathrm{ha})$ & 4 \\
\hline 45 & Glifosato $1088 \mathrm{~g} / \mathrm{ha}(200 \mathrm{l} / \mathrm{ha})$ & 2 y 4 & 29 & Paraquat $500 \mathrm{~g} / \mathrm{ha}(355 \mathrm{l} / \mathrm{ha})$ & 3 \\
\hline \multirow[t]{16}{*}{50} & Paraquat $500 \mathrm{~g} / \mathrm{ha}(300 \mathrm{l} / \mathrm{ha})$ & 1 y 3 & 30 & Glifosato $1088 \mathrm{~g} / \mathrm{ha}(166 \mathrm{l} / \mathrm{ha})$ & \\
\hline & & & & sin parcela de escorrentía & 2 \\
\hline & & & 32 & Deshierba manual de Ipomoea sp. & todas \\
\hline & & & 33 & Chapia con motoguadaña & 3 \\
\hline & & & 35 & Chapia con motoguadaña & 1 \\
\hline & & & 36 & Paraquat $560 \mathrm{~g} / \mathrm{ha}(355 \mathrm{l} / \mathrm{ha})$ & 1 y 3 \\
\hline & & & 37 & Glifosato $1360 \mathrm{~g} / \mathrm{ha}(1661 / \mathrm{ha})$ & \\
\hline & & & & sólo en parcela escorrentía & 2 \\
\hline & & & 38 & Chapia con cuchillo & 4 \\
\hline & & & 38 & Glifosato $1360 \mathrm{~g} / \mathrm{ha}(166 \mathrm{l} / \mathrm{ha})$ & 4 \\
\hline & & & 41 & Chapia con motogüadaña & 3 \\
\hline & & & 44 & Glifosato $1088 \mathrm{~g} / \mathrm{ha}(166 \mathrm{l} / \mathrm{ha})$ & \\
\hline & & & & sin parcela escorrentía & 2 \\
\hline & & & 44 & Chapia motoguadaña & 1 \\
\hline & & & 45 & Paraquat $560 \mathrm{~g} / \mathrm{ha}(355 \mathrm{l} / \mathrm{ha})$ & 1 \\
\hline & & & 46 & Chapia con motogüadaña & 3 \\
\hline
\end{tabular}




\section{Volumen de agua y peso del suelo (sedimentos) presente en los colectores:}

El agua presente en cada colector de $100 \mathrm{~L}$-colocados al final de cada parcela de escorrentía, precedidos por un embudo construido igualmente con láminas galvanizadas- se midió dos o tres veces por semana, se desechó y se dejó que se acumularan los sedimentos durante un periodo de un mes dentro del colector; posteriormente se secaron y pesaron.

\section{Cantidad de nutrimentos presentes en el agua y en los sedimentos:}

Se realizaron análisis de sedimentos y agua trimestralmente, al mezclar las muestras de tres meses; luego se determinó un promedio mensual. Las muestras de sedimentos y agua fueron analizadas según la metodología descrita por Henríquez et al. (1995).

\section{Rendimiento de cada parcela:}

Luego de un muestreo en el área en estudio, se estimó el rendimiento de las parcelas en evaluación, para las cosechas del 2002-2003 y 2003-2004, utilizando la metodología desarrollada por la Beneficiadora Santa Eduviges, dueña de la finca.

\section{RESULTADOS}

\section{Control de malezas}

En general, se observó una disminución del porcentaje de control en las parcelas tratadas con paraquat a lo largo del tiempo, en ambos años. En las primeras semanas del año, se observó un porcentaje de control entre el 90 y $100 \%$, debido a que durante la época seca todas las malezas murieron y el suelo permaneció desnudo. Con las primeras aplicaciones de paraquat se lograron porcentajes de control de hasta un $80 \%$, pero conforme se realizaron más de estas aplicaciones se fueron seleccionando las malezas tolerantes al herbicida y el control fue por lo general inferior al $80 \%$. Este fenómeno se observó más claramente durante el año 2002 (Figura 1 y 2). En el año 2003, al aplicarse medidas alternativas de control, como la "chapia química" (chapia con motoguadaña seguida de aplicación del herbicida), se logró aumentar el porcentaje de control y llegar al $80 \%$ o incluso superarlo en la parcela 1 (Figura 3 ).

En las parcelas tratadas con glifosato se dio un comportamiento similar durante el 2002 -disminución del porcentaje de control en el tiempo- (Figura 1 y 2). Sin embargo, en el 2003 se logró un excelente control en la parcela \#2, debido probablemente a un banco de semillas reducido, y en la parcela \#4 el control fue sumamente difícil debido a la selección de malezas Rubiáceas tolerantes. En esta misma parcela, luego de realizar la chapia con cuchillo y aplicar una dosis mayor de glifosato, se logró un excelente control de estas malezas (Figura 4).

El disturbio ocasionado al suelo y la incorporación de materia orgánica en las parcelas 3 (paraquat) y 4 (glifosato) en la semana 16 del año 2003, atrasó el crecimiento de las malezas, por lo que la aplicación de los herbicidas no fue necesaria sino hasta siete semanas después de realizada esta labor. Sin embargo, la composición florística de estas parcelas no varió significativamente ni tuvo un efecto importante en el control de las malezas posteriormente.
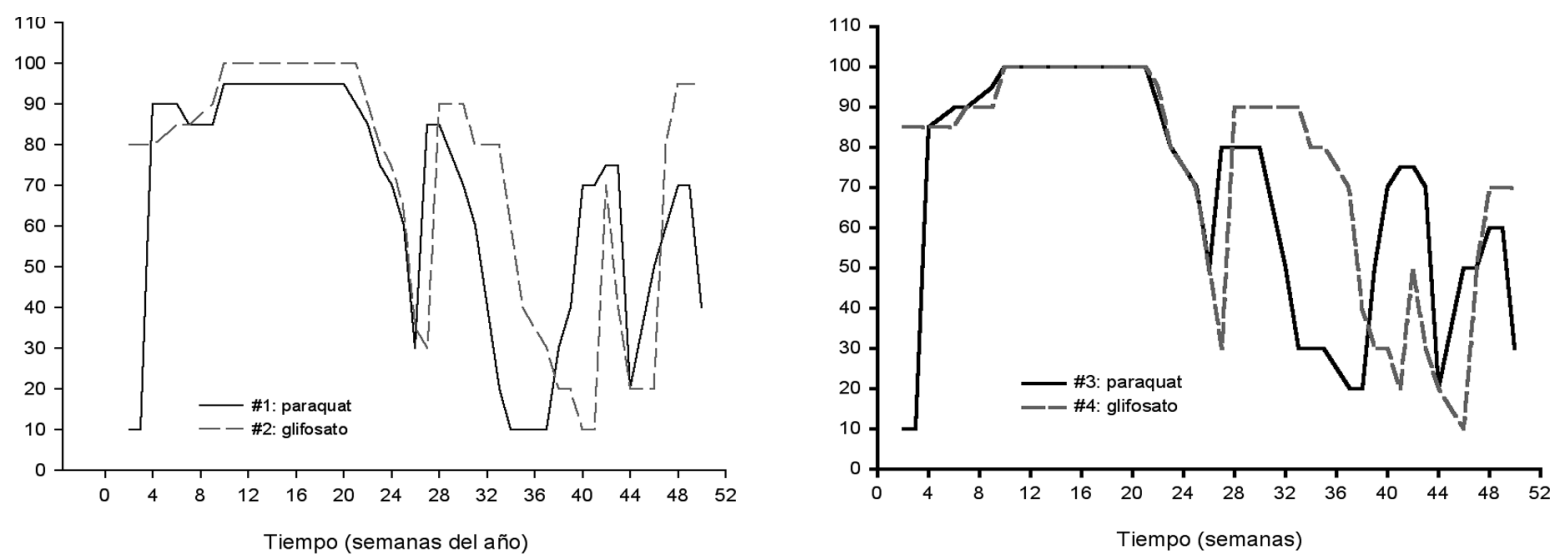

Figura 1 y 2. Porcentaje de control de malezas en general durante el año 2002 en las cuatro parcelas de café tratadas con paraquat y glifosato. San Isidro, Alajuela, Costa Rica. 

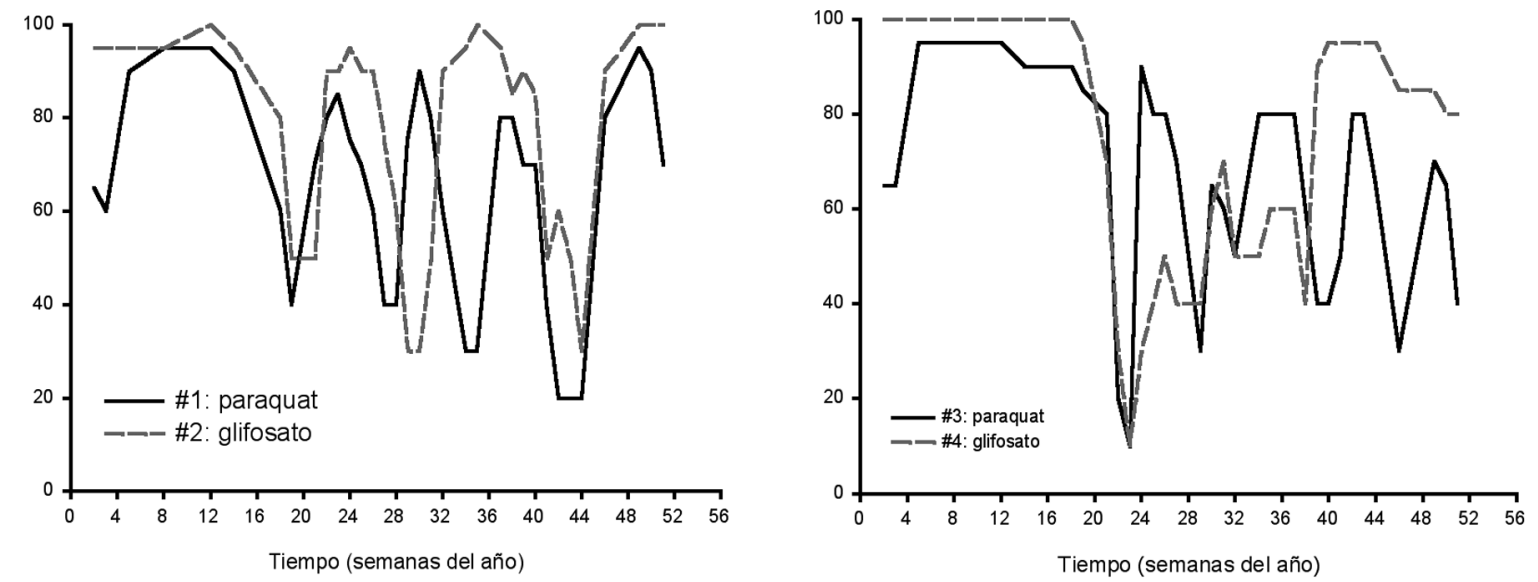

Figura 3 y 4. Porcentaje de control de malezas en general durante el año 2003. Las parcelas 3 y 4 fueron disturbadas en la semana 16. San Isidro, Alajuela, Costa Rica.

\section{Dinámica de poblaciones.}

\section{Parcelas tratadas con paraquat}

Se observó en la parcela 1 una disminución en frecuencia y cobertura de varias malezas a través del tiempo mientras otras especies, que no se encontraban presentes o no eran importantes al inicio del ensayo, aumentaron su IFC. Esto se debe a que al utilizarse una sola molécula herbicida y no rotar productos se seleccionan aquellas malezas que resulten tolerantes o resistentes a ese herbicida. Es por esto que se presentó un incremento en el IFC de Hydrocotyle bowlesiodes a través del tiempo, al punto de convertirse en una de las especies con mayor cobertura y de más difícil control en esta parcela (Cuadro 2). En el caso de Gnaphalium $s p$. , su IFC disminuyó a partir de la semana 33 del 2002 y se mantuvo casi sin variación en el 2003, producto probablemente de las chapias que se realizaron para controlarla. Sin embargo, mantuvo su importancia en cuanto a frecuencia y cobertura. Por otro lado, Cardamine flaccida, que al inicio del ensayo era la maleza de mayor cobertura, fue poco a poco disminuyendo su IFC a través del tiempo, y aun cuando se encontró siempre presente en las parcelas, su importancia también disminuyó. Con el tiempo también se observó un aumento en el IFC de las plantas de café, ya que fueron creciendo y las bandolas cerrando espacios libres. En el 2003 se determinó la aparición y el aumento en el tiempo del IFC de Drimaria cordata, seleccionada por el herbicida.

En la parcela 3 (Cuadro 3) la maleza más importante fue Gnaphalium sp., cuya frecuencia fue alta en toda la parcela y aumentó con el tiempo. Es interesante notar la desaparición de Commelina difussa de esta par- cela, así como la disminución de la importancia de $C$. flaccida en el 2003.

\section{Parcelas tratadas con glifosato}

En las parcelas tratadas con glifosato la especie Cardamine flaccida fue una de las más frecuentes y con mayor cobertura. Sin embargo, debido a su tamaño reducido no es difícil de controlar con ambos herbicidas, y mas bien se podría pensar en su función como cobertura noble en el sistema. Por otro lado, Spermacoce laevis, en la parcela 2, y S. latifolia y S. laevis en la parcela 4 resultaron malezas difíciles de controlar con el glifosato, es por esto que su IFC aumentó paulatinamente (Cuadros 4 y 5). Es interesante notar que Commelina diffusa, si bien es de difícil control con glifosato, disminuyó su IFC con relación al inicio del ensayo, cuando fue una de las malezas más importantes de estas parcelas.

\section{Sedimentos recolectados de las parcelas de esco- rrentía}

En el Cuadro 6 se presentan las características de suelo más importantes para el presente estudio, determinadas a inicios del 2002, al inicio del ensayo. Es importante resaltar las diferencias que se presentaron entre parcelas, lo cual pudo afectar el comportamiento del agua en el suelo y por lo tanto la cantidad de sedimentos que se recogieron en los colectores. El hecho de encontrar variaciones en áreas muy cercanas, en suelos tropicales, ha sido reconocido por diferentes autores tanto dentro como fuera de Costa Rica (Cervantes y Günter 1992). 
Cuadro 2. Índice de frecuencia cobertura (por semana del año) de las malezas presentes en la parcela \#1, tratada con paraquat. San Isidro de Alajuela, Costa Rica. 2002 y 2003.

\begin{tabular}{|c|c|c|c|c|c|c|c|c|c|}
\hline \multirow{3}{*}{ Especie } & \multicolumn{9}{|c|}{ Indice de Frecuencia Cobertura (IFC) } \\
\hline & \multicolumn{5}{|c|}{2002} & \multicolumn{4}{|c|}{2003} \\
\hline & Nov. 2001 & Semana 25 & 33 & 45 & 50 & 20 & 28 & 42 & 44 \\
\hline Amaranthus dubius & - & 0,23 & - & - & - & - & - & - & - \\
\hline Bidens pilosa & 0,11 & 6,87 & 6,18 & 0,24 & - & 0,57 & 0,84 & 0,06 & 0,16 \\
\hline Cardamine flaccida & 47,3 & 43,5 & 22,3 & 20,63 & 0,07 & & 5,06 & 0,06 & 2,12 \\
\hline Coffea arabica & - & - & - & 18,73 & 38,58 & 19,66 & 28,69 & 39,31 & 33,14 \\
\hline Commelina difussa & 2,86 & - & 0,12 & - & - & - & - & - & - \\
\hline Conyza sp. & 0,11 & - & - & 0,32 & - & 2,85 & & 3,02 & 1,65 \\
\hline Cynodon nfluensis & - & - & - & - & - & 32,48 & - & - & - \\
\hline Cyperus ferax & - & 4,58 & 1,45 & - & 0,07 & - & - & - & - \\
\hline Digitaria $s p$ & - & 0,38 & - & - & - & - & - & - & - \\
\hline Drimaria cordata & - & - & - & - & - & - & 1,94 & 14,34 & 10,35 \\
\hline Eleusine indica & 1,76 & - & - & - & - & - & - & - & - \\
\hline Galinsoga parviflora & 0,66 & 0,23 & 4,36 & 2,86 & 1,05 & 0,34 & - & - & \\
\hline Gnaphalium sp. & - & - & 32,12 & 13,49 & 9,36 & 2,85 & 12,15 & 17,30 & 16,67 \\
\hline Hidrocotyle bowlesiodes & 2,31 & - & 0,97 & 3,02 & 20,45 & 1,14 & 5,74 & 9,62 & 19,45 \\
\hline Ipomoea purpurea & - & - & 0,61 & - & - & - & 0,42 & 1,01 & 1,73 \\
\hline Oxalis sp. & 0,22 & - & - & - & - & - & - & - & - \\
\hline Paspalum paniculatum & - & - & - & - & 1,65 & - & - & - & 0,04 \\
\hline Portulaca oleracea & - & 2,75 & 0,73 & - & - & 10,83 & - & - & - \\
\hline Richardia scabra & 3,96 & 2,44 & - & 1,9 & - & - & - & - & - \\
\hline Solanum nigrum & - & - & - & 0,56 & - & - & - & - & - \\
\hline Sonchus oleracea & 1,76 & 8,78 & 1,45 & 0,48 & 0,67 & - & - & - & - \\
\hline Spermacoce laevis & 9,67 & 4,58 & 1,82 & - & - & 0,57 & 0,25 & - & - \\
\hline Spermacoce latifolia & & & & & & & 1,35 & 0,13 & 0,20 \\
\hline Spermacoce ocimoides & - & - & - & - & - & - & 0,25 & - & - \\
\hline
\end{tabular}

Cuadro 3. Índice de frecuencia cobertura (según semana del año) de las malezas presentes en la parcela \#3, tratada con paraquat. San Isidro de Alajuela, Costa Rica. 2002 y 2003.

\begin{tabular}{|c|c|c|c|c|c|c|c|c|}
\hline \multirow{3}{*}{ Especie } & \multicolumn{8}{|c|}{ Indice de Frecuencia Cobertura (IFC) } \\
\hline & \multicolumn{5}{|c|}{2002} & \multicolumn{3}{|c|}{2003} \\
\hline & Nov. 2001 & Semana 25 & 33 & 45 & 50 & 23 & 29 & 41 \\
\hline Amaranthus dubius & 0,24 & - & 0,6 & - & - & & & \\
\hline Bidens pilosa & - & 0,66 & 0,75 & 0,65 & 2,29 & 0,13 & 0,32 & 0,06 \\
\hline Cardamine flaccida & 15,98 & 21,05 & 23,88 & 44,86 & - & 25,63 & 5,12 & 0,12 \\
\hline Coffea arabica & - & - & - & 25,23 & 63,43 & 50,31 & 44,20 & 66,37 \\
\hline Commelina difussa & 4,26 & 0,13 & 0,9 & - & - & & & \\
\hline Conyza sp. & 0,118 & - & - & 2,24 & $-3,89$ & 0,06 & & 1,05 \\
\hline Cynodon nfluensis & & & & & & 1,38 & 3,77 & 0,18 \\
\hline Euphorbia hirta & 0,36 & - & - & - & - & & & \\
\hline Galinsoga parviflora & 1,18 & 0,13 & 0,15 & - & - & 5,50 & 0,22 & \\
\hline Gnaphalium $s p$ & 1,18 & 3,20 & 4,26 & 14,95 & 10,06 & & 13,37 & 22,46 \\
\hline Hidrocotyle bowlesioides & 0,83 & - & - & - & - & & & \\
\hline Ipomoea purpurea & 3,2 & 1,32 & 1,5 & 0,19 & 0,23 & 0,25 & 1,46 & 0,18 \\
\hline Kylinga brevifolia & & & & & & & & 0,12 \\
\hline Paspalum paniculatum & - & 12,11 & 2,69 & - & 0,57 & & & \\
\hline Portulaca oleracea & 10,3 & 18,16 & 20,6 & 2,25 & 0,69 & 0,94 & 0,16 & \\
\hline Richardia scabra & - & 0,13 & - & 1,12 & - & & & \\
\hline Sida sp. & 0,24 & - & - & - & - & & & \\
\hline Sonchus oleracea & 0,13 & - & 0,75 & 0,09 & 0,11 & 0,25 & 1,29 & \\
\hline Spermacoce alata & 1,18 & 0,13 & 0,3 & - & - & & & \\
\hline Spermacoce laevis & 0,24 & - & - & - & 0,46 & 1,13 & 0,97 & \\
\hline Spermacoce latifolia & & & & & & & & 0,12 \\
\hline Spermacoce ocimoides & & & & & & & 0,11 & \\
\hline
\end{tabular}


Cuadro 4. Índice de frecuencia cobertura (por semana del año) de las malezas presentes en la parcela \#2, tratada con glifosato. San Isidro de Alajuela, Costa Rica. 2002 y 2003.

\begin{tabular}{|c|c|c|c|c|c|c|}
\hline \multirow{3}{*}{ Especie } & \multicolumn{6}{|c|}{$\begin{array}{l}\text { Indice de Frecuencia Cobertura } \\
\text { (IFC) }\end{array}$} \\
\hline & \multicolumn{4}{|c|}{2002} & \multicolumn{2}{|c|}{2003} \\
\hline & $\begin{array}{l}\text { Nov. } \\
2001\end{array}$ & $\begin{array}{c}\text { Semana } \\
25\end{array}$ & 37 & 45 & 20 & 44 \\
\hline Amaranthus dubius & 0,83 & - & 0,7 & - & & \\
\hline Bidens pilosa & 0,56 & 10,35 & 2,5 & 2,1 & 4,44 & \\
\hline Cardamine flaccida & 31,11 & 49,65 & 1,2 & 39,5 & & 8,26 \\
\hline Coffea arabica & - & - & 4,7 & 4,38 & 62,42 & 74,03 \\
\hline Commelina diffusa & 2,04 & - & - & 0,1 & & \\
\hline Conyza sp. - & - & 2,5 & - & & 9,09 & \\
\hline Cyperus ferax & - & - & 4,7 & 0,38 & & \\
\hline Digitaria sp. & & & & & & 0,05 \\
\hline Galinsoga parviflora & & & & & 3,23 & \\
\hline Gnaphalium sp. & 0,37 & - & 4,7 & - & & \\
\hline Hipochoeris radicata & 0,185 & 2,52 & - & - & & \\
\hline Ipomoea purpurea & 2,04 & - & & - & 3,03 & 2,03 \\
\hline Papalum paniculatum & - & - & 0,4 & 0,58 & & \\
\hline Portulaca oleracea & 0,28 & 0,98 & 2,8 & 0,38 & 3,64 & \\
\hline Richardia scabra & - & 1,4 & 0,6 & 4 & & \\
\hline Sida $s p$ & 0,09 & - & - & - & & \\
\hline Sonchus oleracea & - & 1,96 & 5,6 & 1,14 & & \\
\hline Spermacoce laevis & 3,33 & 0,56 & 1 & 25,2 & 0,40 & 1,04 \\
\hline Spermacoce latifolia & - & 0,7 & 3,3 & - & & 1,19 \\
\hline Spermacoce ocimoides & - & - & 7,3 & - & 0,40 & \\
\hline
\end{tabular}

Cuadro 5. Índice de frecuencia cobertura (según semana del año) de las malezas presentes en la parcela \#4, tratada con glifosato. San Isidro de Alajuela, Costa Rica. 2002 y 2003.

\begin{tabular}{|c|c|c|c|c|c|c|}
\hline \multirow{3}{*}{ Especie } & \multicolumn{6}{|c|}{$\begin{array}{l}\text { Indice de Frecuencia Cobertura } \\
\text { (IFC) }\end{array}$} \\
\hline & \multicolumn{4}{|c|}{2002} & \multicolumn{2}{|c|}{2003} \\
\hline & $\begin{array}{l}\text { Nov. } \\
2001\end{array}$ & $\begin{array}{l}\text { Semana } \\
25\end{array}$ & 37 & 45 & 23 & 28 \\
\hline Bidens pilosa & & & & & 0,38 & \\
\hline Cardamine flaccida & 18,63 & 72,5 & - & 37,6 & 25,38 & 10,04 \\
\hline Coffea arabica & - & - & - & 2,21 & 48,46 & 55,72 \\
\hline Commelina diffusa & 26,56 & 1,6 & 10,9 & 2,36 & & \\
\hline Conyza sp.0,08 & - & - & - & & & \\
\hline Cyperus ferax & - & - & 0,36 & - & 0,23 & \\
\hline Digitaria sp. & & & & & 0,08 & \\
\hline Eleusine indica & 0,31 & - & - & - & & \\
\hline Galinsoga parviflora & 0,08 & - & 0,24 & - & 22,31 & \\
\hline Gnaphalium sp. & 0,15 & - & 2,18 & - & & \\
\hline Ipomoea purpurea & - & 1,83 & 0,24 & - & & \\
\hline Kilinga vermifolia & - & - & 0,12 & - & & \\
\hline Paspalum paniculatum & 4,28 & - & - & 0,3 & & \\
\hline Portulaca oleracea & 6,41 & 5,74 & 0,85 & - & 0,08 & \\
\hline Richardia scabra & - & - & 0,73 & - & & \\
\hline Sida sp. $\quad 0,38$ & - & - & - & & & \\
\hline Solanum nugrum & - & - & 2,91 & - & & \\
\hline Sonchus oleracea & - & - & 0,24 & - & & \\
\hline Spermacoce alata & 2,44 & - & 5,09 & 7,08 & & 9,74 \\
\hline Spermacoce latifolia & - & - & 20 & 30,7 & & \\
\hline Spermacoce ocimoides & - & - & 8 & - & & 15,06 \\
\hline Urtica sp. & & & & & & 0,15 \\
\hline
\end{tabular}

Cuadro 6. Características químicas y físicas de las parcelas en estudio. San Isidro, Alajuela, Costa Rica. 2002.

\begin{tabular}{|c|c|c|c|c|c|c|c|c|c|c|}
\hline \multirow{3}{*}{ Parcela } & \multicolumn{9}{|c|}{ Nutrimentos } & \multirow[b]{3}{*}{$\% \mathrm{MO}$} \\
\hline & $\mathrm{pH}\left(\mathrm{H}_{2} \mathrm{O}\right)$ & \multicolumn{3}{|c|}{$\operatorname{cmol}(+) / L$} & \multicolumn{5}{|c|}{$\mathrm{mg} / \mathrm{L}$} & \\
\hline & & $\mathbf{C a}$ & Mg & $\mathbf{K}$ & $\mathbf{P}$ & $\mathrm{Cu}$ & $\mathbf{F e}$ & Mn & $\mathbf{Z n}$ & \\
\hline 1 (paraquat) & 4,85 & 2,10 & 0,83 & 0,25 & 21,10 & 35,00 & 26,00 & 45,00 & 2,00 & 7,76 \\
\hline 2 (glifosato) & 4,70 & 2,74 & 0,99 & 0,25 & 15,30 & 52,00 & 31,00 & 54,00 & 1,50 & 7,57 \\
\hline 3 (paraquat) & 4,80 & 3,04 & 0,73 & 0,43 & 23,60 & 50,00 & 37,00 & 51,00 & 1,20 & 5,27 \\
\hline 4 (glifosato) & 4,55 & 2,60 & 0,77 & 0,24 & 23,50 & 74,00 & 48,00 & 55,00 & 1,00 & 7,07 \\
\hline
\end{tabular}

Textura (muestra de $0-30 \mathrm{~cm}$ de profundidad)

\begin{tabular}{crrrl}
\hline Parcela & Arena & Limo & Arcilla & Nombre textural \\
\hline 1 (paraquat) & 42,3 & 41,8 & 16,0 & Franco \\
2 (glifosato) & 39,8 & 36,4 & 23,8 & Franco \\
3 (paraquat) & 32,3 & 33,9 & 33,8 & Franco-Arcilloso \\
4 (glifosato) & 58,1 & 26,0 & 16,0 & Franco-Arenoso
\end{tabular}

\begin{tabular}{ccccc}
\hline \multicolumn{2}{c}{ Parcela } & \multicolumn{2}{c}{ Textura en parcelas de escorrentía } & \multicolumn{2}{c}{ Pendientes } \\
& Ap & \multicolumn{1}{c}{ Bw } & Parcela & Pendiente \\
\hline 1 (paraquat) & $0-55 \mathrm{~cm}$, franco & $55-120 \mathrm{~cm}$, franco arcilloso & 1 & $25 \%$ \\
2 (glifosato) & $0-18 \mathrm{~cm}$, franco & $18-120 \mathrm{~cm}$, franco arcilloso & 2 & $27 \%$ \\
3 (paraquat) & $0-13 \mathrm{~cm}$, franco & $13-50+\mathrm{cm}$, franco & 3 & $27 \%$ \\
4 (glifosato) & $0-15 \mathrm{~cm}$, franco & $15-50+\mathrm{cm}$, arcilloso & 4 & $30 \%$ \\
\hline
\end{tabular}


Entre las características que afectan mayormente la dinámica hídrica en estas parcelas está la textura. Así, mientras la parcela 3 (paraquat) es de textura franco arcilloso y por lo tanto más susceptible al lavado por el agua de escorrentía, la parcela 4 (glifosato) presenta textura franco arenosa, lo que en principio indicaría una mayor capacidad de infiltración. Por otro lado, los contenidos de materia orgánica de ambas parcelas también permiten predecir una menor unión de los agregados del suelo de la parcela 3 al tener un menor porcentaje de materia orgánica.

Asimismo, el horizonte Ap de la parcela 1 tiene una profundidad tres veces mayor al mismo horizonte de las demás parcelas, lo que le confiere una capacidad de infiltración mayor.

Analizando las pendientes de estas parcelas, existe una diferencia de $5 \%$ entre la parcela 1 y la 4 , por lo que probablemente el agua que escurre en esta última lo hará con mayor velocidad al haber mayor inclinación del terreno; sin embargo, también influye la rugosidad del mismo, característica que no se determinó. Chaplot y Le Bissonnais (2003) encontraron correlación entre la intensidad de la lluvia y la longitud e inclinación de la pendiente en la mayor o menor pérdida de suelo por erosión.

\section{III.1. Cantidad de sedimentos depositados en los colectores}

En el año 2002 se erosionaron de las parcelas tratadas con paraquat $2,71 \mathrm{~kg}$ de suelo en promedio, en un área de $14 \mathrm{~m}^{2}$ (1,94 t/ha), si se mantuvieran las mismas condiciones de suelo y ambientales en toda el área (Cuadro 7). Por su parte, de las parcelas tratadas con glifosato se erosionaron $5,54 \mathrm{~kg}$ en promedio, ó 3,96 t/ha.
Para el año 2003 se obtuvieron valores muy diferentes con respecto al año anterior. Esta variación en los resultados de erosión en años diferentes también ha sido reportada por Aristizábal (1987) en cafetales de Colombia.

Debido a que las parcelas 3 y 4 fueron disturbadas, sólo es posible hacer comparaciones entre ellas mismas en los dos años en estudio. Así, se aprecia una notable disminución en la cantidad de suelo erosionado en el año 2003 con respecto al año 2002. Esto se puede asociar a dos factores: primero, al realizar la incorporación de materia orgánica se mejoraron características de estructura del suelo como adhesividad entre los agregados, lo que disminuyó el efecto separador de las gotas de lluvia; segundo, el comportamiento de las lluvias en este año fue significativamente diferente al año anterior en cuanto a su distribución a lo largo del año. Esto pudo haber significado menor duración y/o intensidad de los aguaceros y por lo tanto menor erosión en la parcelas.

Tomando los datos obtenidos en estas dos parcelas, se podría decir que, bajo condiciones similares de suelo y ambientales, se habrían erosionado $170,5 \mathrm{~kg} / \mathrm{ha} / \mathrm{a}-$ ño utilizando paraquat y $187 \mathrm{~kg} / \mathrm{ha}$ con glifosato, en suelo disturbado.

En las parcelas sin disturbar, la erosión sería de 231 $\mathrm{kg} / \mathrm{ha} / \mathrm{año}$ aplicando paraquat y $329 \mathrm{~kg} / \mathrm{ha}$ con glifosato.

\section{III.3. Nutrimentos presentes en los sedimentos}

Los elementos que se pierden más fácilmente del suelo en el agua de escorrentía, debido a su alta solubilidad en ésta, son el calcio, magnesio y potasio potasio (Schaefer et al. 2002; Bertol et al. 2003). Por otro lado,

Cuadro 7. Peso (g) mensual de los sedimentos acumulados en los colectores de las parcelas de escorrentía. San Isidro, Alajuela, Costa Rica. 2002 y 2003.

\begin{tabular}{cccccccccccccccc}
\hline Parcela & \multicolumn{2}{c}{ Ene-May } & \multicolumn{2}{c}{ Jun } & \multicolumn{2}{c}{ Jul-Ago } & \multicolumn{2}{c}{ Set } & Oct & & Nov & Totales \\
\hline & 2002 & 2003 & 2002 & 2003 & 2002 & 2003 & 2002 & 2003 & 2002 & 2003 & 2002 & 2003 & 2002 & 2003 \\
1 & 233,73 & 102,50 & 20,26 & 109,40 & 28,87 & 52,00 & $72,00 *$ & 19,40 & 254,00 & 24,80 & 97,00 & 15,20 & 739,31 & 323,30 \\
2 & 68,78 & 274,30 & 16,02 & 62,70 & 42,34 & 54,00 & $130,00^{*}$ & 23,00 & 110,00 & 31,60 & 50,00 & 14,80 & 429,11 & 460,40 \\
3 & 360,24 & 41,80 & 22,63 & 101,40 & 29,47 & 55,00 & 837,00 & 10,00 & 477,00 & 3,50 & 225,00 & 27,00 & 1972,20 & 238,70 \\
4 & 1137,20 & 59,60 & 174,40 & 87,20 & 175,84 & 92,30 & 3113,00 & $* *$ & 369,00 & 12,20 & 107,00 & 10,60 & 5109,73 & 261,90 \\
\hline
\end{tabular}

Debe considerarse que los datos de las parcelas 3 y 4 del año 2003 corresponden a parcelas que fueron disturbadas en mayo de ese año

*Datos incompletos por pérdida de sedimentos

**Datos no obtenidos por pérdida del material

Parcelas 1 y 3: paraquat, Parcelas 2 y 4: glifosato 
la materia orgánica cumple en el suelo un papel importante como adhesivo que une los agregados del suelo, haciendo que el salpique de la lluvia no los separe fácilmente y sean arrastrado por el agua que escurre por la superficie del suelo.

En el año 2002, las parcelas tratadas con paraquat y glisofato presentaron, en promedio, una pérdida de bases en cantidades importantes, tomando en cuenta el rango adecuado de estos elementos en el suelo. Comparativamente, la cantidad de calcio, magnesio y potasio que se perdió de las parcelas con escorrentía en los sedimentos fue muy similar en ambos tratamientos (Cuadro 8). Sin embargo, debido a que en las parcelas aplicadas con glifosato se erosionó una mayor cantidad de suelo, se perdieron una mayor cantidad de bases junto con ese suelo en comparación con las parcelas tratadas con paraquat. Durante el año 2003 se determinó en ambos tratamientos un aumento en la pérdida de calcio y magnesio tanto en las parcelas sin disturbar como en las parcelas disturbadas en comparación con el año anterior, y al igual que en el 2002, estas cantidades consti-

Cuadro 8. Cantidad promedio de bases presentes en los sedimentos extraídos de las parcelas de escorrentía. San Isidro de Alajuela, Costa Rica. 2002 y 2003.

\begin{tabular}{lrrrrrr}
\hline & \multicolumn{6}{c}{$\mathbf{c m o l}(+) / \mathbf{L}$} \\
\cline { 2 - 7 } Parcela & $\mathbf{2} \mathbf{C a}$ & \multicolumn{2}{c}{$\mathbf{M g}$} & \multicolumn{2}{c}{$\mathbf{K}$} \\
& $\mathbf{2 0 0 2}$ & $\mathbf{2 0 0 3}$ & $\mathbf{2 0 0 2}$ & $\mathbf{2 0 0 3}$ & $\mathbf{2 0 0 2}$ & $\mathbf{2 0 0 3}$ \\
\hline 1 (paraquat) & 5,91 & 10,20 & 1,16 & 1,96 & 0,78 & 0,70 \\
2 (glifosato) & 7,70 & 8,38 & 1,55 & 2,02 & 0,83 & 0,72 \\
3 (paraquat) & 8,70 & $\mathbf{1 0 , 1 2}$ & 1,52 & $\mathbf{1 , 9 2}$ & 0,70 & $\mathbf{0 , 6 4}$ \\
4 (glifosato) & 6,67 & $\mathbf{7 , 7 3}$ & 1,59 & $\mathbf{1 , 6 1}$ & 0,55 & $\mathbf{0 , 7 4}$ \\
\hline
\end{tabular}

Debe considerarse que los datos de las parcelas 3 y 4 del año 2003 corresponden a parcelas que fueron disturbadas en mayo de ese año. tuyen salidas importantes de nutrimentos del suelo. En ambos años se perdió una cantidad importante de potasio de las cuatro parcelas, tomando en cuenta que el rango óptimo de esta base en el suelo es de 0,2-0,8. En los sedimentos recogidos, el nivel de este elemento se aproxima al máximo. Estos resultados son similares a los reportados por varios autores (Schaefer et al. 2002; Bertol et al. 2003).

\section{Volumen de agua recolectado de las parcelas de escorrentía}

\section{IV.1. Volúmenes de agua presente en los colectores}

Como se muestra en el Cuadro 9, se pudo determinar que no existe relación entre los volúmenes de agua recogidos en los colectores y la cantidad de sedimentos arrastrados hacia éstos. Es decir, mientras que en la parcela 1 (paraquat) durante el 2002 se recogieron 700,8 1 de agua y 739,31 g de sedimentos, al año siguiente 1435,93 1 de agua arrastraron tan solo 323,3 g de suelo. Esto quiere decir que la cantidad de suelo que se pierde no depende en mayor medida del volumen de agua de escorrentía, sino de otros factores como la intensidad de la lluvia que golpea directamente en el suelo y por supuesto de las características del suelo de cada parcela. Además, el mayor crecimiento de las plantas de café hace que las gotas no caigan directamente sobre el suelo, reduciéndose su efecto en la erosión.

\section{IV.2: Nutrimentos presentes en el agua recolectada}

Para el año 2002, considerando los datos de 1/ha de agua de escorrentía calculados anteriormente, junto con los valores de nutrimentos en el agua de escorrentía de cada parcela (Cuadro 10), se puede determinar que, en promedio, en el agua de escorrentía proveniente de las

Cuadro 9. Volumen (1) mensual de agua recogida en los colectores de las parcelas de escorrentía. San Isidro, Alajuela, Costa Rica. 2002 y 2003 .

\begin{tabular}{cccccccccccccccc}
\hline Parcela & \multicolumn{1}{c}{ Ene-May } & \multicolumn{2}{c}{ Jun } & \multicolumn{2}{c}{ Jul-Ago } & \multicolumn{2}{c}{ Set } & Oct & & Nov & Totales \\
\hline & 2002 & 2003 & 2002 & 2003 & 2002 & 2003 & 2002 & 2003 & 2002 & 2003 & 2002 & 2003 & 2002 & 2003 \\
1 & 187,0 & 107,7 & 21,5 & 465,7 & 81,2 & 137,9 & 42,8 & 192,7 & 192,9 & 256,4 & 138,4 & 275,6 & 700,8 & 1435,9 \\
2 & 137,6 & 199,7 & 48,1 & 426,2 & 115,7 & 212,1 & 92,0 & 196,2 & 229,3 & 310,2 & 113,6 & 291,6 & 758,7 & 1635,9 \\
3 & 205,9 & $\mathbf{3 8 , 4}$ & 46,7 & $\mathbf{3 1 1 , 7}$ & 138,8 & $\mathbf{6 2 , 7}$ & 173,7 & $\mathbf{1 6 4 , 3}$ & 602,9 & $\mathbf{3 6 1 , 2}$ & 202,4 & $\mathbf{5 5 2 , 7}$ & 1393,5 & $\mathbf{1 4 9 0 , 9}$ \\
4 & 204,9 & $\mathbf{8 1 , 8}$ & 102,7 & $\mathbf{3 0 1 , 7}$ & 183,3 & $\mathbf{1 1 3 , 0}$ & 275,3 & 168,9 & 627,1 & $\mathbf{2 5 3 , 8}$ & 252,9 & $\mathbf{2 9 5 , 4}$ & 1680,3 & $\mathbf{1 2 1 4 , 6}$ \\
\hline
\end{tabular}

Debe considerarse que los datos de las parcelas 3 y 4 del año 2003 corresponden a parcelas que fueron disturbadas en mayo de ese año Parcelas 1 y 3: paraquat, Parcelas 2 y 4: glifosato 
Cuadro 10. Cantidad promedio $(\mathrm{mg} / \mathrm{L})$ de nutrimentos presentes en el agua recolectada proveniente de las parcelas de escorrentía. San Isidro, Alajuela, 2002.

\begin{tabular}{cccccccccccccc}
\hline Parcela & \multicolumn{2}{c}{$\mathbf{N}-\mathbf{~ N H 4}$} & \multicolumn{2}{c}{$\mathbf{N}-\mathbf{~ N O 3}$} & \multicolumn{2}{c}{ Ca } & \multicolumn{2}{c}{ Mg } & \multicolumn{2}{c}{ K } & \multicolumn{2}{c}{$\mathbf{P}$} \\
\hline & 2002 & 2003 & 2002 & 2003 & 2002 & 2003 & 2002 & 2003 & 2002 & 2003 & 2002 & 2003 \\
\hline 1 (paraquat) & 0,26 & 0,59 & 8,76 & 4,38 & 45,96 & 0,02 & 3,1 & 0,15 & 8,33 & 0,12 & 2,00 & 0,7 \\
2 (glifosato) & 0,01 & 1,98 & 4,40 & 5,08 & 44,1 & 0,04 & 3,03 & 0,03 & 5,07 & 0,4 & 1,55 & 0,47 \\
3 (paraquat) & 0,38 & $\mathbf{0 , 7 7}$ & 9,00 & $\mathbf{2 , 5 8}$ & 46,66 & $\mathbf{0 , 0 2}$ & 3,3 & $\mathbf{0 , 0 0 7}$ & 6,57 & $\mathbf{0 , 4 2}$ & 1,48 & $\mathbf{0 , 4 7}$ \\
4 (glifosato) & 0,24 & $\mathbf{0 , 5 8}$ & 8,32 & $\mathbf{2 , 3 8}$ & 42,9 & $\mathbf{0 , 0 2}$ & 2,9 & $\mathbf{0 , 0 1}$ & 6,96 & $\mathbf{0 , 2 5}$ & 1,48 & $\mathbf{0 , 4 7}$ \\
\hline
\end{tabular}

parcelas tratadas con paraquat se perdieron $46,31 \mathrm{mg} / \mathrm{l}$ de calcio, esto es $34,6 \mathrm{~kg} / \mathrm{ha}$. En las parcelas aplicadas con glifosato se determinó una pérdida promedio de $43,5 \mathrm{mg} / \mathrm{l}$ de calcio ó $37,8 \mathrm{~kg} / \mathrm{ha}$

En general, en las parcelas correspondientes al tratamiento con paraquat se perdieron mayores cantidades de nutrimentos en el agua de escorrentía con respecto a las parcelas de glifosato. Bertol et al. (2003) explican este fenómeno indicando que al disminuir la cantidad de material erosionado, éste estará compuesto por partículas más pequeñas, lo que resulta en un aumento en la proporción de coloides minerales. El Ca y $\mathrm{Mg}$ son adsorbidos fuertemente por estos coloides en suspensión y por lo tanto su pérdida será elevada.

Durante el año 2003, la pérdida de nutrimentos en el agua de escorrentía fue considerablemente menor que el año anterior, en todas las parcelas. Los valores obtenidos son muy similares entre todas las parcelas, incluyendo a las parcelas disturbadas. Se puede decir que esta pérdida no es significativa.

\section{Rendimiento por parcela}

Según se observa en el Cuadro 11, los rendimientos estimados de las parcelas tratadas con glifosato fueron mayores, en promedio, en el año 2002 a los obteni-

Cuadro 11. Rendimientos estimados de café en las parcelas tratadas con paraquat y glifosato. San Isidro, Alajuela, Costa Rica. 2002-2003.

\begin{tabular}{ccc}
\hline \multirow{2}{*}{ Tratamiento } & \multicolumn{2}{c}{ Fanegas/manzana } \\
\cline { 2 - 3 } & $\mathbf{2 0 0 2}$ & $\mathbf{2 0 0 3}$ \\
\hline & & \\
Parcela 1 (paraquat) & 24,50 & 44,48 \\
Parcela 2 (glifosato) & 48,66 & 45,93 \\
Parcela 3 (paraquat) & 35,71 & 34,27 \\
Parcela 4 (glifosato) & 24,42 & 35,15 \\
\hline
\end{tabular}

dos en las parcelas aplicadas con paraquat. En el año 2003 los rendimientos entre ambos tratamientos no difieren significativamente. Al ser el café una planta bianual, se podría pensar que el efecto de los tratamientos, aplicados en el año 2002, se representa de una mejor manera en el rendimiento del año 2003.

\section{CONCLUSIONES}

Producto de las aplicaciones de un solo herbicida en cada parcela evaluada, se seleccionaron aquellas especies tolerantes a cada herbicida, de manera que resultó cada vez más difícil su control. Se realizaron durante los dos años once aplicaciones de paraquat y nueve de glifosato, además de siete chapias en las parcelas correspondientes a paraquat y una en parcela de glifosato. Esto significa un mayor costo económico del control de malezas con paraquat con respecto al uso del glifosato. Sin embargo, este control se hace cada vez menos eficiente y conlleva el riesgo del desarrollo de resistencia por parte de las malezas hacia los herbicidas empleados, por lo que se hace necesaria la escogencia de diferentes herbicidas que puedan rotarse y usarse en diferentes periodos de tiempo. El estudio de dinámica de poblaciones mostró la desaparición de malezas que antes de iniciar el experimento dominaban el sitio, pero que fueron desplazadas por aquellas malezas que los herbicidas empleados fueron seleccionando incluso a partir de la tercera aplicación.

Por otro lado, se observó una mayor pérdida de suelo y un mayor volumen de agua de escorrentía en las parcelas tratadas con glifosato, y por lo tanto una mayor pérdida de nutrimentos al representar mayores volúmenes que los obtenidos de la parcelas aplicadas con paraquat. Es importante tener en cuenta que estas pérdidas de suelo, agua y nutrimentos no se pueden asociar solamente a las aplicaciones de estos herbicidas, ya que las características físicas y químicas del suelo de cada una de las parcelas son distintas entre sí e influyen en mayor o menor medida en el fenómeno de erosión. Aun 
así, los resultados obtenidos evidencian un efecto del herbicida empleado en la pérdida de suelo de las parcelas de escorrentía.

\section{LITERATURA CITADA}

AGUILAR, V.; STAVER, C.; MILBERG, P. 2003. Weed vegetation response to chemical and manual selective ground cover management in a shaded coffee plantation. Weed Research, 43:68-73.

ARISTIZÁBAL, A. 1987. Manejo y control integrado de malezas en el cultivo del café y la erosión de los suelos. In: IV Congreso Colombiano de la Ciencia del Suelo. CENICAFE. Colombia. p. 25.

BARBIER, EB.; BISHOP, JT. 1995. Economic values and incentives affecting soil and water conservation in development countries. J. Soil and Water Cons. 50:133-139.

BERTOL, I.; LEMOS, E.; GUADAGNIN, JC.; VEDANA, AL.; CARRAFA, MR. 2003. Nutrient losses by water erosion. Scientia Agricola, 60:581-586.

BERTSCH, F. 1995. La fertilidad de los suelos y su manejo. Asociación Costarricense de la Ciencia del Suelo. San José, Costa Rica. 157 p.

CAMPER, N.D (ed.). 1986. Research methods in weed science. Southern Weed Science Society. Illinois. p. 37.

CERVANTES, C.; GÜNTER, W. 1992. Características físicas y pérdida de nutrimentos de las parcelas de erosión de Cerbatana de Puriscal, Costa Rica. Agronomía Costarricense 16(1): 99-106.

CHAPLOT, VA.; Le BISSONNAIS, Y. 2003. Runoff features for interrill erosion at different rainfall intensities, slope lenghts, and gradient in an agricultural loessial hillslope. Soil Sci. Soc. Am. J. 67:844 -851.

COGO, NP.; LEVIEN, R.; SCHWARZ, RA. 2003. Perdas de solo e agua por erosao hídrica influenciadas por métodos de preparo, classes de declive e niveis de fertilidade do solo. R. Bras. Ci. Solo. 27:743-753

HANSEN, LT.; BRENEMAN, VE.; DAVISON, CW.; DICKEN, CW. 2002. The cost of soil erosion to downstream navigation. J. Soil and Water Cons. 57: 205-213.

HENRÍQUEZ, C.; BERTSCH, F.; SALAS, R. 1995. Fertilidad de suelos: Manual de Laboratorio. Asociación Costarricense de la Ciencia del Suelo (ACCS). San José. pp. $35-47$.

JÜRGENS, G. 1983. Levantamiento de malezas en cultivos agrícolas. In: Koch, W.; Helmut, W.; Sauerborn, J. (eds.). Resúmenes del Seminario Manejo Integrado de Malezas. PLITS 3(2) 1985. Stuttgart. pp. 85-104.

LAL, R. 1990. Soil erosion in the tropics, principles and management. Estados Unidos. Mac Graw Hill, 1990. 580 p.

NEARING, MA.; PRUSKI, EF.; O`NEAL, MR. 2004. Expected climate change impacts on soil erosion rates: A review. J. Soil and Water Cons. 59:43-51.

REYNAUD, CA.; SILVA, DD.; NERI, KW.; FALCO, F.; ALBUQUERQUE, MR.; ABREU, M. 2002. Perdas de solo, nutrientes, materia orgánica e efeitos microestruturais em Argissolo Vermelho-Amarelo sob chuva simulada. Pesq. Agropec. Bras. 37:669-678.

VENCILL, W.K. (ed.). 2002. Herbicide Handbook. Weed Science Society of America (WSSA), Kansas. 493 p. 